\title{
Ablation of microstructures applying diffractive elements and UV femtosecond laser pulses
}

\author{
J.J.J. Kaakkunen · J. Bekesi · J. Ihlemann · P. Simon
}

Received: 10 November 2009 / Accepted: 10 May 2010 / Published online: 10 June 2010

(C) Springer-Verlag 2010

\begin{abstract}
A new method for simple and economic fabrication of diffractive optical elements (DOEs) with three and four phase levels, by UV nanosecond (ns) laser ablation is presented. The technique is based on the combination of two sequentially generated complementary 2-level phase elements. During the fabrication, complete ablative removal of a highly absorbing silicon suboxide layer by pixelated backside illumination ensures the necessary high precision and optical quality. Full functionality of the new DOEs is demonstrated by fabricating micro-structures using UV femtosecond pulses.
\end{abstract}

\section{Introduction}

There is a rapidly increasing interest in the generation of micro- and nano-size structures in numerous scientific and industrial applications [1-4]. Such patterns can be used to modify light's propagation, beam shape, and polarization properties [5]. It has also been shown that specially designed structures might have various functionalities such as hydrophobic or hydrophilic behaviors [6], field amplification capability, increased absorbance, etc. [7, 8]. The steadily increasing number of applications generates an ever increasing demand on simple, fast, and economical fabrication methods.

J.J.J. Kaakkunen $(\bowtie)$

Department of Physics and Mathematics, University of Joensuu,

Yliopistonkatu 7, POBOX 111, 80100 Joensuu, Finland

e-mail: jarno.kaakkunen@joensuu.fi

Fax: +49-551-503599

J. Bekesi $\cdot$ J. Ihlemann $\cdot$ P. Simon

Laser-Laboratorium Göttingen e.V., Hans-Adolf-Krebs-Weg 1,

37077 Göttingen, Germany
In previous works, we investigated a simple and low-cost method for generating phase masks as diffractive optical elements (DOEs) on fused silica substrates for UV laser applications. The mask fabrication process is based on backside ablation of a silicon suboxide $\left(\mathrm{SiO}_{x}\right)$ layer on a bulk quartz substrate [9]. This $\mathrm{SiO}_{x}$ layer has a significantly increased absorption in the UV, allowing to overcome the well-known fabrication difficulties of standard fused silica [10]. After ablating the desired pattern through backside illumination, the remaining $\mathrm{SiO}_{x}$ layer can easily be converted into $\mathrm{SiO}_{2}$ in an annealing process [9, 11-13]. An unpleasant drawback of this technique is its limitation to only two phase levels. Backside ablation ensures the creation of smooth ablated features with perfect optical quality and exact depth control, necessary to create highly efficient phase elements. However, this process also prohibits the simple generation of more than two phase levels.

In the present article, we propose a new method to overcome this limitation. It is based on the combination of two properly designed and sized complementary 2-level phase elements, whose combined effect results in a 3- or 4-level DOE. In case of a 2-level phase element, the generated diffraction pattern is always symmetric to the optical axis, and suppression of the -1 st-order is not possible. This limits the theoretically achievable maximum efficiency to considerably less than $50 \%$. Only very special point-symmetrical patterns can be designed in the on-axis mode (the zeroth order and the diffracted orders propagate into the same direction), where the 0th-order cannot be separated, but efficiencies around $80 \%$ or more can be achieved. In all other cases, an off-axis design has to be applied allowing to separate the 0th-order; however, imposing strong limitations on the efficiency. On the contrary, applying more than 2 phase levels, the 1st-order can be suppressed, thus increasing the theoretically achievable efficiency to well over 50\%. Addi- 
tionally, increasing the number of phase levels leads to the creation of cleaner and smoother intensity distributions, and a better control over scattered light. Fabrication of 3- and 4-level beam shaping elements is demonstrated using the newly proposed method. Different kinds of on-axis and offaxis designs have been realized, characterized, and tested in material processing experiments.

\section{Design concept}

The basic idea to overcome the problem of limited number of levels is to find a method to separate the multilevel phase mask designs into two-level systems, that take advantage of the fabrication process based on the $\mathrm{SiO}_{x}$ layer ablation (to date applicable for 2-level phase elements only), and finally synthesize them to a multilevel system. The principle of a possible separation is shown in Fig. 1 for 3-and 4-level elements.

The top part of Fig. 1a shows all possible phase steps of a 3 -level phase mask. Levels 1, 2, and 3 introduce $0,2 \pi / 3$, and $4 \pi / 3$ phase shifts, respectively. The lower part shows two 2-level phase masks, which are contacted with their structured sides so that they together provide 3 phase levels. In the 3-level case, both components have the same step size, introducing either 0 or $2 \pi / 3$ phase shifts. By proper positioning of the steps, the same phase shifts can be achieved as in the case of the original 3-level element. Level 1 can be simulated by introducing 0 phase shifts on both mask components. Simulating level 2 a phase shift of $2 \pi / 3$ should be introduced by, e.g. on the upper mask and 0 on the lower one or vice versa. For the 3 rd level, both mask components have to introduce a phase shift of $2 \pi / 3$, causing an overall shift of $4 \pi / 3$. The same separation principle can also be used to generate 4-level phase elements (Fig. 1b), with the only difference, that is in that case 4 different levels have to be generated with phase shifts of $0, \pi / 2, \pi$, and $3 \pi / 2$ (top part of the figure). This can only be managed if two different step sizes (in practice two different $\mathrm{SiO}_{x}$ layer thicknesses) are applied (lower part of the figure), where the upper mask (Mask 1) has a layer thickness of $h$ and the lower one (Mask 2) a thickness of $2 h$.

The required height of the steps $\left(h_{k}\right)$ can easily be calculated by (1), where $h_{k}$ defines the height of the steps on the substrate material for the different levels. Here, $\lambda$ is the designed illumination wavelength, $n_{1}$ is the refractive index of the substrate material at the illumination wavelength, $n_{2}$ is the refractive index of the air at $\lambda, L$ is the number of levels on the mask (e.g. $L=3$ for 3 levels), and $\mathrm{k}$ is the level index (e.g $k=1, \ldots, 3$ for 3 phase levels),

$h_{k}=\frac{\lambda}{n_{1}-n_{2}} \frac{(L-k)}{L}, \quad k=1, \ldots, L$.

Before fabrication the designed multilevel DOE phase maps have to be separated according to the method discussed above. In the computer design of the phase map, the different phase levels are marked with gray levels. Black is used to mark the pixels with 0 phase shift and by increasing shift the color turns to gray and is getting lighter and lighter. A two-level mask is represented by black and white, and a 4-level map has 4 different gray levels: black (level 1 in Fig. 1b), dark gray (level 2), light gray (level 3) and white (level 4), respectively. Separation of this 4-level mask into two 2-level ones means, we have to generate two phase maps with only black and white pixels. Referring to Fig. 1, the gray levels of the 4-level mask have to be manipulated so that for Mask 1 dark gray is converted to white and light gray to black, meanwhile the original colors black and white stay unchanged. For Mask 2, dark gray is converted to black and light gray to white. A part of a 4-level mask and
Fig. 1 Transformation of a 3-level and b 4-level DOE data into two complementary 2-level DOE systems

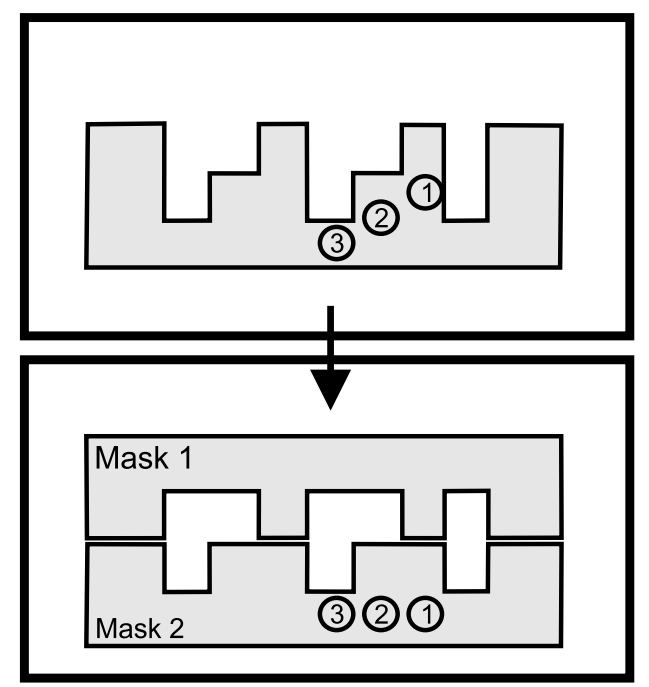

a 3-level

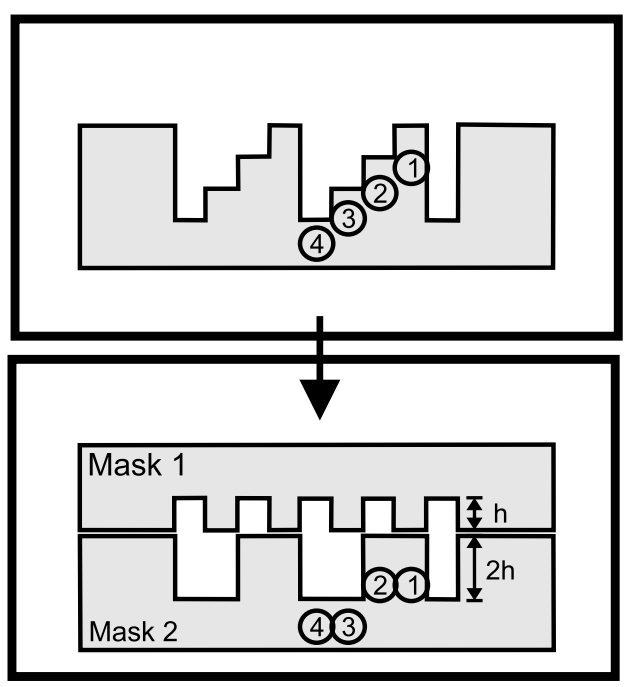

b 4-level 


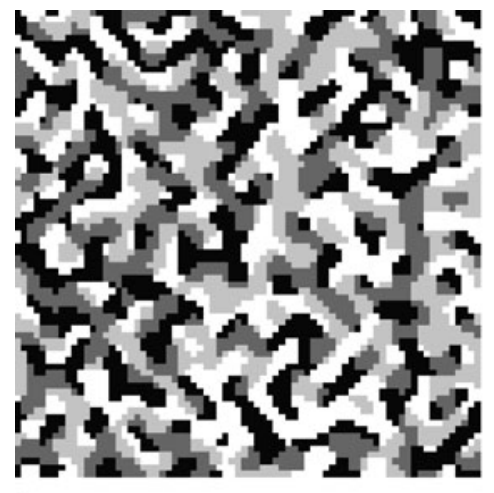

a

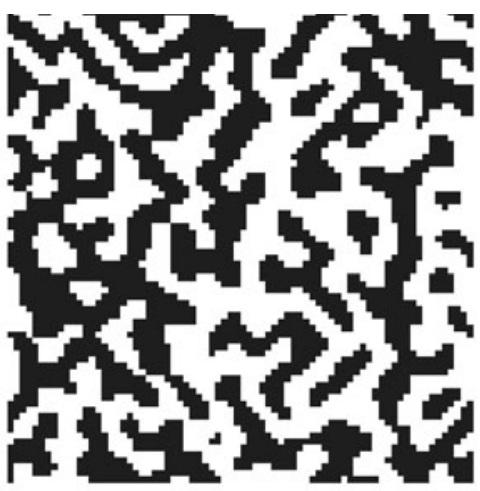

b

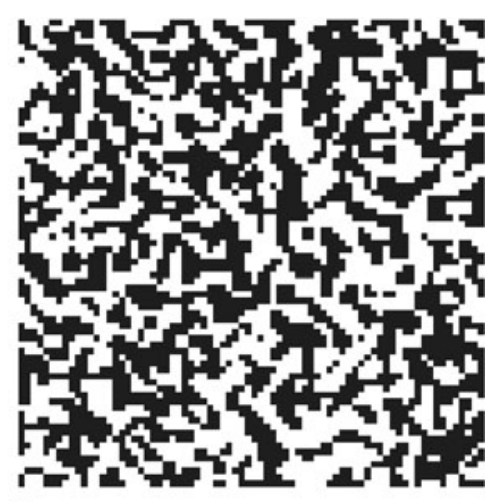

c

Fig. 2 Original 4-level data of an on-axis DOE (a), and transformed data into complementary systems of b $248 \mathrm{~nm}(\pi)$ and $(\mathbf{c}) 124 \mathrm{~nm}(\pi / 2)$ step sizes

its corresponding converted versions Mask 1 and Mask 2 are displayed in Fig. 2.

We used an Iterative Fourier Transform Algorithm (IFTA) to optimize our DOEs [1]. The masks were designed for an illumination wavelength of $\lambda=248 \mathrm{~nm}$. The pixel size was $27 \mu \mathrm{m}$, and the number of pixels was $367 \times 367$. The free aperture size of the DOEs was around $10 \mathrm{~mm} \times 10 \mathrm{~mm}$. The resulting diffraction intensity pattern was a $25 \times 25$ dot-matrix, generated either on the optical axis of the system (on-axis design), or with a lateral displacement (off-axis design). First, we designed three- and four-level on-axis elements that provide $25 \times 25$ dot-matrix distributions, so that the generated pattern is coaxial with the 0th-order beam. In the Fourier plane, the 0th-order is focused in a spot, whose position and size is just the same as that of the central dot of the 1st-order dot-matrix. In a following step, we also designed a 4-level off-axis DOE, providing the same dotmatrix intensity distribution as that of the on-axis ones.

\section{Fabrication and alignment}

For the machining of the phase masks, we used a commercial excimer laser (Lambda Physik LPX 300) delivering UVnanosecond laser pulses at a wavelength of $\lambda=193 \mathrm{~nm}$. To generate the required phase steps, the pixels were ablated in the $\mathrm{SiO}_{x}$-layer by imaging a square aperture onto the backside of the quartz substrate. In Fig. 3a microscope image of one of the ablated 2-level $\mathrm{SiO}_{x}$-layers is shown, which was generated through the separation process. After fabrication and annealing, the 2-level masks have to be faced together and aligned, building up the required 3- or 4-level phase map. It has been shown that even small alignment errors can cause degradations in the diffraction efficiency and the pattern shape, and usually it increases the stray light content of the zeroth order [14]. The alignment process is challenging but the necessary precision can be achieved by mounting one of the masks on a 4-axis $(x, y, z, \varphi)$ positioning stage, and applying a simple monitoring system, consisting of an illuminating source, a camera objective, and a CCD-camera. To reach a good overlap, a lateral positioning precision of around $1 \mu \mathrm{m}$ is needed (for a pixel size of $27 \mu \mathrm{m}$ ), the rotational precision should be better than $0.1 \mathrm{mrad}$ (resulting in a maximal displacement of $1 \mu \mathrm{m}$ over $10 \mathrm{~mm}$, which was the typical aperture size of our phase elements). Figure $3 b$ shows the image of the aligned complementary 2-level mask assembly of a three-level design, viewed in transmission by our monitoring system. It can be seen that very good alignment of the masks can be achieved with almost perfect overlap of the pixels.

\section{Characterization and application}

In order to test the feasibility of the new approach, the diffraction patterns generated by the DOEs were first evaluated by a UV sensitive camera system. Femtosecond laser pulses at a wavelength of $248 \mathrm{~nm}$ [15] were used to illuminate the DOEs whose diffraction pattern was analyzed in the Fourierplane (far-field). The obtained distributions for the 3- and 4-level on-axis elements are displayed in Fig. 4. In the case of the 3-level, DOE imperfect suppression of the 0th-order is visible. In contrast, an almost complete vanishing central spot for the 4-level DOE can be seen (Fig. 4b). This effect is not fully understood. According to the theoretical calculations, a fairly uniform distribution over all spots (including the central one) is expected, even if complete suppression of the 0th-order is achieved. The measured uniformity of the diffraction pattern was somewhat poorer than that predicted by the theoretical calculations. This might be caused by fabrication errors of the DOEs and because of residual alignment errors of the complementary elements. The theoretically calculated efficiency of the 3-level and 4-level DOEs were $67 \%$ and $73 \%$, respectively, the corresponding measured efficiencies were $60 \%$ in both cases. 
Fig. 3 Light microscope image of the ablated $\mathrm{SiO}_{x}$ layer and b picture of the carefully aligned complementary systems forming a 3-level DOE, viewed in transmission
Fig. 4 Far-field images of the a 3- and b 4-level on-axis elements

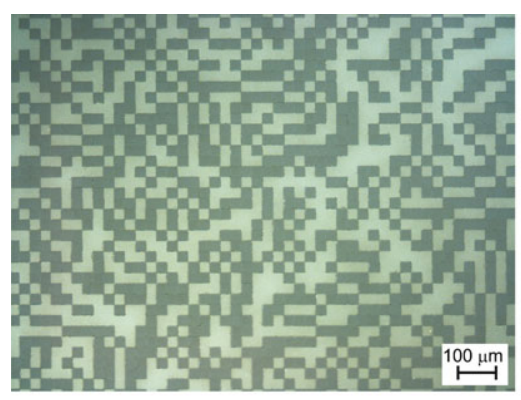

a

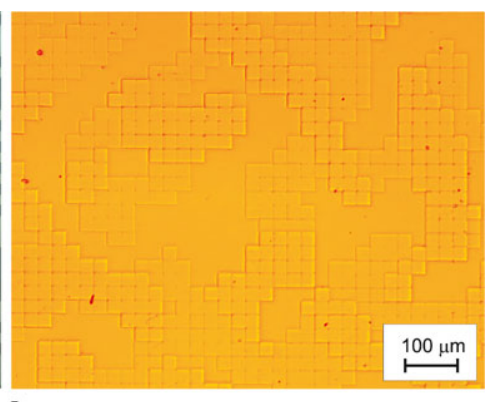

b

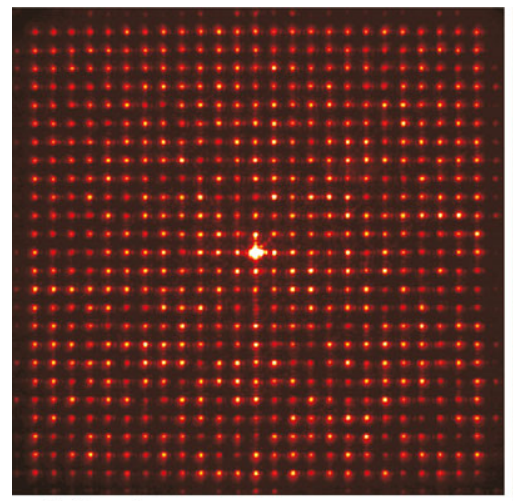

a 3-level

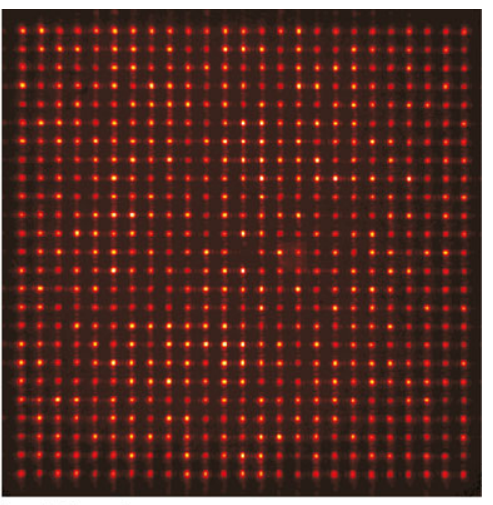

b 4-level
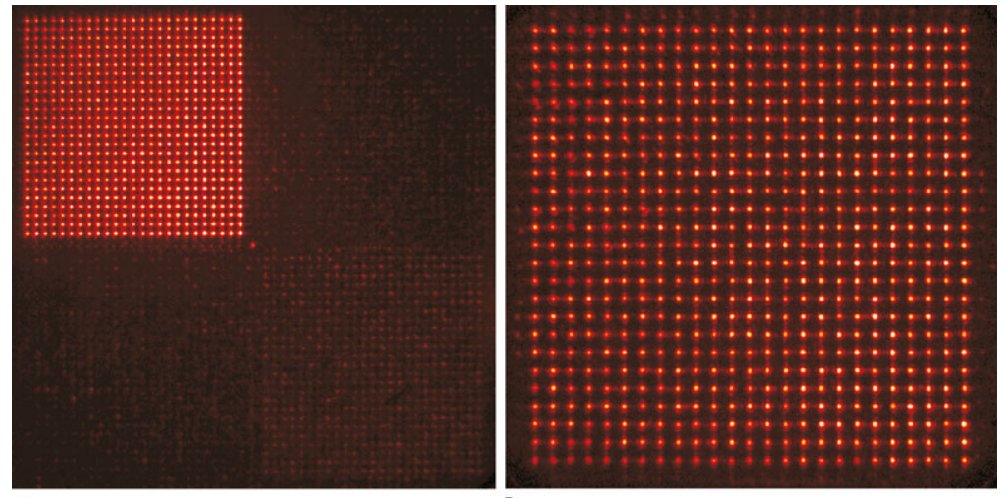

a

b
Fig. 5 a Far-field image of the 4-level off-axis element. b A close up view of the more intense +1 -order.
Additionally, we fabricated a four-level off-axis DOE using the same method. In Fig. 5a, a CCD-camera image of the far-field diffraction pattern obtained by this element is shown. As Fig. 5a shows, the diffraction efficiency of the +1 -order is notably higher than that of the -1 -order. This is in good agreement with the results of theoretical calculations, predicting a diffraction efficiency of $56 \%$ for the +1 diffraction order, and only few percent for the -1 -order. The measured diffraction efficiency values of these orders were found to be $43 \%$ for +1 -order and $3 \%$ for -1 -order. It is also notable that in the case of the 4-level DOE the shape and uniformity of the spots are also better than that of the on-axis ones.

Finally, the 4-level off-axis element was tested in an ablation experiment as a proof of its applicability for high res- olution material processing. In order to reach a high quality and background-free intensity pattern, a filtering element has been applied in the Fourier-plane to reduce noise and scattered light. For this purpose, first a slightly misaligned telescope was used to generate an enlarged diffraction pattern of the DOE with an overall size of a few $\mathrm{mm}$. Then a filtering element consisting of a two-dimensional array of $25 \times 25$ holes was placed there. The holes were large enough not to block the spots, but filtering the residual background irradiation between them. Finally, this plane was imaged onto the sample surface. In Fig. 6, light microscope images of such ablated patterns are shown. The applied total pulse energy was $0.55 \mathrm{~mJ}$ and the applied pulse number was 200 . Here, the pitch of the holes was $15.5 \mu \mathrm{m}$ with an average hole diameter of $14 \mu \mathrm{m}$. This image shows a high quality 
Fig. 6 Ablations pattern of polyimide (PI) using the novel off-axis 4-level DOE device, obtained with 200 pulses, displayed with two different magnifications

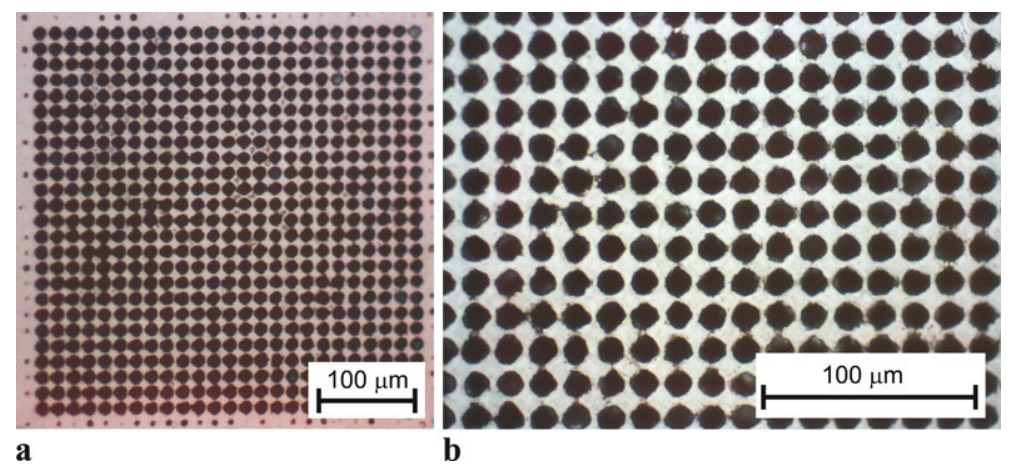

a b ablated pattern without any remarkable damage between the dots. Such elements might be used to create high aspect ratio holes.

\section{Conclusions}

We have demonstrated the feasibility of generating threeand four-level diffractive optical phase elements by using $\mathrm{SiO}_{x}$ backside ablation in combination with a novel technique, based on the separation of multilevel designs into complementary 2-level system. Different types of on-axis and off-axis designs for creating 3- and 4-level DOEs have been realized and tested. The presented technique is suitable for the simple and economic fabrication of high quality DOEs. Using the new devices in material processing experiments, it has been shown that high quality ablated structures can be achieved.

Acknowledgements This work was supported by TEKES, the Finnish Agency for Technology and Innovation.

\section{References}

1. J. Turunen, F. Wyrowski, Diffractive Optics for Industrial and Commercial Applications (Akademie Verlag, Berlin, 1997)

2. M.J. Thomson, M.R. Taghizadeh, J. Mod. Opt. 50, 1691-1699 (2003)

3. G.P. Behrmann, M.T. Duignan, Appl. Opt. 36, 4666-4674 (1997)

4. R.J. Winfield, M. Meister, G.M. Crean, S. Paineau, Mater. Sci. Semicond. Process. 3, 481-486 (2000)

5. H.-P. Herzig, Micro-Optics: Elements, Systems and Applications (Taylor \& Francis, London, 1997)

6. H.Y. Erbil, A.L. Demirel, Y. Avci, O. Mert, Science 299, 1377 (2003)

7. J.J.J. Kaakkunen, K. Paivasaari, M. Kuittinen, T. Jaaskelainen, Appl. Phys. A, Mater. Sci. Process. 94, 215-220 (2009)

8. B. Bhushan, Y.C. Jung, Ultramicroscopy 107, 1033 (2007)

9. M. Schulz-Ruhtenberg, J. Ihlemann, J. Heber, Appl. Surf. Sci. 587, 190 (2005)

10. J. Ihlemann, M. Schulz-Ruhtenberg, T. Fricke-Begemann, J. Phys., Conf. Ser. 59, 206-209 (2007)

11. J. Bekesi, D. Schäfer, J. Ihlemann, P. Simon, SPIE 4977, 235 (2003)

12. J. Ihlemann, J. Optoelectron. Adv. Mater. 7, 1191-1195 (2005)

13. J. Ihlemann, D. Schäfer, Appl. Surf. Sci. 197-198, 856-861 (2002)

14. A.J. Caley, M. Braun, A.J. Waddie, M.R. Taghizadeh, Appl. Opt. 46, 2180-2188 (2007)

15. S. Szatmari, F.P. Schäfer, Opt. Commun. 68, 196 (1988) 